\title{
Tensor Network Renormalization
}

\author{
G. Evenbly ${ }^{1, *}$ and G. Vidal ${ }^{2,}$ \\ ${ }^{1}$ Institute for Quantum Information and Matter, California Institute of Technology, Pasadena California 91125, USA \\ ${ }^{2}$ Perimeter Institute for Theoretical Physics, Waterloo, Ontario N2L 2Y5, Canada
}

(Received 21 April 2015; published 29 October 2015)

\begin{abstract}
We introduce a coarse-graining transformation for tensor networks that can be applied to study both the partition function of a classical statistical system and the Euclidean path integral of a quantum many-body system. The scheme is based upon the insertion of optimized unitary and isometric tensors (disentanglers and isometries) into the tensor network and has, as its key feature, the ability to remove short-range entanglement or correlations at each coarse-graining step. Removal of short-range entanglement results in scale invariance being explicitly recovered at criticality. In this way we obtain a proper renormalization group flow (in the space of tensors), one that in particular (i) is computationally sustainable, even for critical systems, and (ii) has the correct structure of fixed points, both at criticality and away from it. We demonstrate the proposed approach in the context of the 2D classical Ising model.
\end{abstract}

DOI: 10.1103/PhysRevLett.115.180405

Understanding emergent phenomena in many-body systems remains one of the major challenges of modern physics. With sufficient knowledge of the microscopic degrees of freedom and their interactions, we can write the partition function of a classical system, namely, a weighted sum of all of its microscopic configurations, or the analogous Euclidean path integral of a quantum many-body system, where the weighted sum is now over all conceivable trajectories. These objects contain complete information on the collective properties of the many-body system. However, evaluating partition functions or Euclidean path integrals is generically very hard. Kadanoff's spin-blocking procedure [1] opened the path to nonperturbative approaches based on coarse graining a lattice [2,3]. More recently, Levin and Nave proposed the tensor renormalization group (TRG) [4], a versatile realspace coarse-graining transformation for 2D classical partition functions - or, equivalently, Euclidean path integrals of 1D quantum systems.

The TRG is an extremely useful approach that has revolutionized how we coarse-grain lattice models [4-9]. However, this method fails to remove part of the shortrange correlations in the partition function and, as a result, the coarse-grained system still contains irrelevant microscopic information. Conceptually, this is in conflict with the very spirit of the renormalization group (RG) and results in a RG flow with the wrong structure of noncritical fixed points, as discussed in Ref. [7]. Computationally, the accumulation of short-range correlations over successive TRG coarse-graining transformation also has important consequences: as pointed out by Levin and Nave, it implies the breakdown of the TRG at criticality [4,10] (although universal information, such as critical exponents, can still be obtained from finite systems).

An analogous problem, faced earlier in the context of ground state wave functions, was resolved with the
PACS numbers: 05.30.-d, 02.70.-c, 03.67.Mn, 75.10.Jm

introduction of entanglement renormalization techniques $[18,19]$. In this Letter we will adapt those techniques to the coarse graining of partition functions or Euclidean path integrals, and we will demonstrate that the resulting scheme generates a proper RG flow, with the correct structure of critical and noncritical fixed points.

A distinctive feature of our proposal, which we call tensor network renormalization (TNR), is that it removes short-range correlations from the partition function at each coarse-graining step. In this way, the effective tensor network description at a given length scale is free from irrelevant microscopic details belonging to shorter length scales. The upshot is a computationally sustainable RG flow in the space of tensors. At criticality, removal of shortrange correlations circumvents the TRG's breakdown, while scale invariance is explicitly realized in the form of a critical fixed-point tensor [20]. This allows us to effectively address infinitely large systems, avoiding finitesize effects. Off criticality, we also obtain the correct structure of noncritical RG fixed points. In this case, the corresponding fixed-point tensors coincide with those previously obtained by Gu and Wen's tensor-entanglementfiltering renormalization (TEFR) $[7,21]$. For simplicity, we demonstrate TNR with the statistical partition function of the 2D classical Ising model (equivalently, the Euclidean path integral of the 1D quantum Ising model with transverse magnetic field), although the key ideas apply to general two-dimensional lattice models and extend to higher dimensions.

Renormalization group flow in the space of tensors.Our starting point, also used in the TRG [4-9], is the observation that the partition function $Z$ of a $2 \mathrm{D}$ classical system (for concreteness assumed to be translation invariant on a periodic square lattice) can be rewritten as a $2 \mathrm{D}$ tensor network made of $N$ copies of some tensor $A$ [see Eq. (5) and Figs. 1(a) and 1(b) for an explicit example], 


$$
Z=\sum_{i j k \ldots} A_{i j k l} A_{m n o j} A_{k r s t} A_{o p q r} \ldots \equiv \operatorname{Tr}\left(\otimes_{x=1}^{N} A\right) .
$$

Here, each index hosts a $\chi$-level local degree of freedom (e.g., $i=1,2, \ldots, \chi$ ), the tensor components $A_{i j k l}$ are local weights, and the tensor trace $t \operatorname{Tr}$ denotes a sum over configurations of all of the indices $i j k \ldots .$.

Our goal is to produce an effective tensor $A^{(1)}$, roughly accounting for four copies of the original tensor $A^{(0)} \equiv A$, such that $Z$ can be approximately expressed as a coarser tensor network made of just $N / 4$ copies of $A^{(1)}$, $Z \approx \operatorname{tTr}\left(\otimes_{x=1}^{N / 4} A^{(1)}\right)$. By iteration, a sequence of tensors

$$
A^{(0)} \rightarrow A^{(1)} \rightarrow A^{(2)} \rightarrow \cdots
$$

will be produced such that, for any length scale $s$,

$$
Z \approx \mathrm{t} \operatorname{Tr}\left(\otimes_{x=1}^{N_{s}} A^{(s)}\right), \quad N_{s} \equiv N / 4^{s} .
$$

Thus, after $\tilde{s} \equiv \log _{4}(N)$ iterations (assuming $N=4^{\tilde{s}}$ for some integer $\tilde{s}>0$ ), the partition function $Z$ becomes the trace of a single tensor $A^{(\tilde{s})}, Z \approx \sum_{i j} A_{i j i j}^{(\tilde{s})}$, which we can finally evaluate [24]. On the other hand, in the thermodynamic limit $N \rightarrow \infty$, we can study the flow in the space of tensors given by Eq. (2). The fixed-point tensors of this flow will capture the universal properties of the phases and phase transitions of the system.

Tensor network renormalization.-Our coarse-graining transformation for the partition function $Z$ in Eq. (1) is based on locally inserting (exact or approximate) resolutions of the identity into the tensor network. The goal is to reorganize the local degrees of freedom, so as to be able to identify and remove those that are only correlated at short distances.

Let us regard each index of the network as hosting a $\chi$-dimensional complex vector space $\mathbb{V} \equiv \mathbb{C}^{\chi}$. We consider two types of insertions; see Fig. 1(c). The first type is implemented by a pair $u u^{\dagger}=I^{\otimes 2}$ of unitary transformations $u$, or disentanglers, acting on two neighboring indices, $u: \mathbb{V} \otimes \mathbb{V} \rightarrow \mathbb{V} \otimes \mathbb{V}$. The disentanglers $u$ will be used to remove short-range correlations [25]. Notice that inserting a pair of disentanglers $u u^{\dagger}$ does not change the partition function $Z$ represented by the tensor network.

The second type of insertion is implemented by a projector $v v^{\dagger}$ (or $w w^{\dagger}$ ), where the isometry $v$ (or $w$ ) combines two indices into a single one, $v: \mathbb{V} \rightarrow \mathbb{V} \otimes \mathbb{V}$, with $v^{\dagger} v=I$. Since $v v^{\dagger}$ is not the identity but a $\chi$-dimensional projector acting on the $\chi^{2}$-dimensional space $\mathbb{V} \otimes \mathbb{V}$, inserting it into the tensor network introduces a truncation error into the representation of the partition function $Z$. This error can be estimated by the norm $\|\delta\|$ of the difference operator $\delta$ defined in Fig. 1(d). If, somehow, only a small truncation error $\|\delta\|$ is introduced, then the resulting tensor network will still be a good approximation to the partition function $Z$.
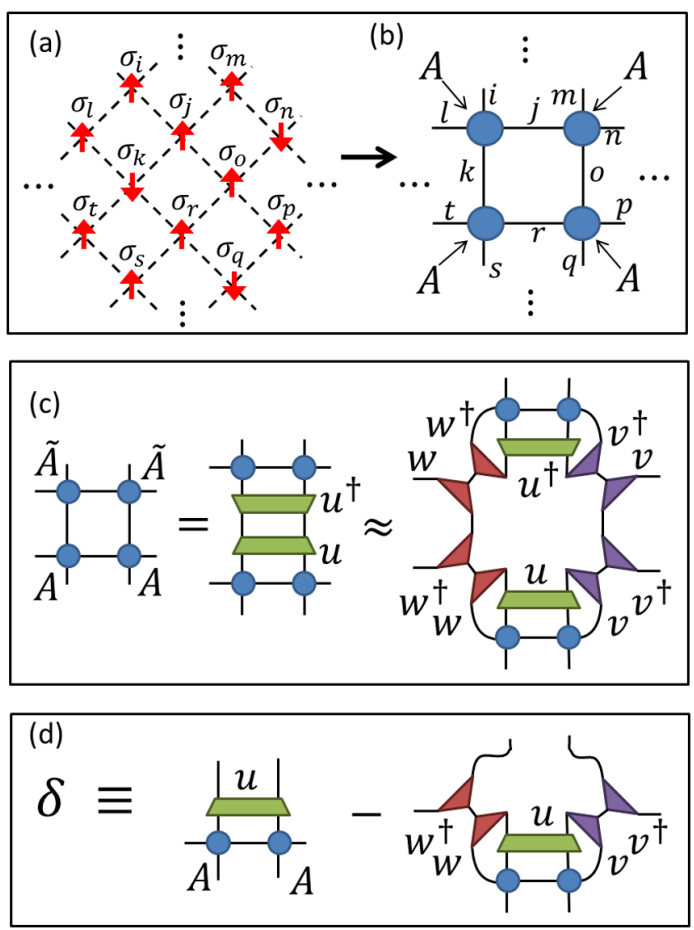

FIG. 1 (color online). (a) As an example, we consider a square lattice (slanted $45^{\circ}$ ) of classical spins, where $\sigma_{k} \in\{+1,-1\}$ is an Ising spin on site $k$. (b) Graphical representation of a part of the tensor network, where each circle denotes a tensor $A$, for the partition function $Z$ of the classical spin model, see Eq. (1). Here, tensor $A_{i j k l}$ encodes the Boltzmann weights of the spins $\left\{\sigma_{i}, \sigma_{j}, \sigma_{k}, \sigma_{l}\right\}$ according to the Hamiltonian function $H$; see Eq. (5). (c) Insertion of a pair of disentanglers $u u^{\dagger}$ between four tensors, where tensors $\tilde{A}$ are obtained from tensors $A$ through a gauge transformation on their horizontal indices [23], followed by an insertion of four projectors of the form $v v^{\dagger}$ (or $w w^{\dagger}$ ). These projectors introduce a truncation error. (d) Tensor $\delta$, whose norm $\|\delta\|$ measures the truncation error introduced by the isometries $v$ and $w$. Disentanglers and isometries are chosen so as to minimize $\|\delta\|$.

Figure 2 shows graphically the proposed TNR transformation. In Fig. 2(a), disentanglers and isometries are inserted between blocks of $2 \times 2$ tensors $A^{(s)}$. In Fig. 2(b) two types of auxiliary tensors, $B^{(s)}$ and $C^{(s)}$, are produced by contracting indices. In Fig. 2(c) tensors $B$ and $C$ are split using a singular value decomposition, as is done in the TRG [26]. Finally, in Fig. 2(d) the coarse-grained tensor $A^{(s+1)}$ at scale $s+1$ is obtained by further contraction of indices. The disentanglers and isometries introduced in Fig. 2(a) are chosen so as to minimize the truncation error $\|\delta\|$ in Fig. 1(d), using well-established, iterative optimization methods for unitary and isometric tensors [19], which are further detailed in Ref. [27]. The overall computational cost of computing tensor $A^{(s+1)}$ from tensor $A^{(s)}$ scales as $O\left(\chi^{7}\right)$, although this cost can be reduced to $O\left(\chi^{6}\right)$ through introducing controlled approximations [27].

To gain some insight into how TNR operates, let us consider first an oversimplified scenario where the partition 


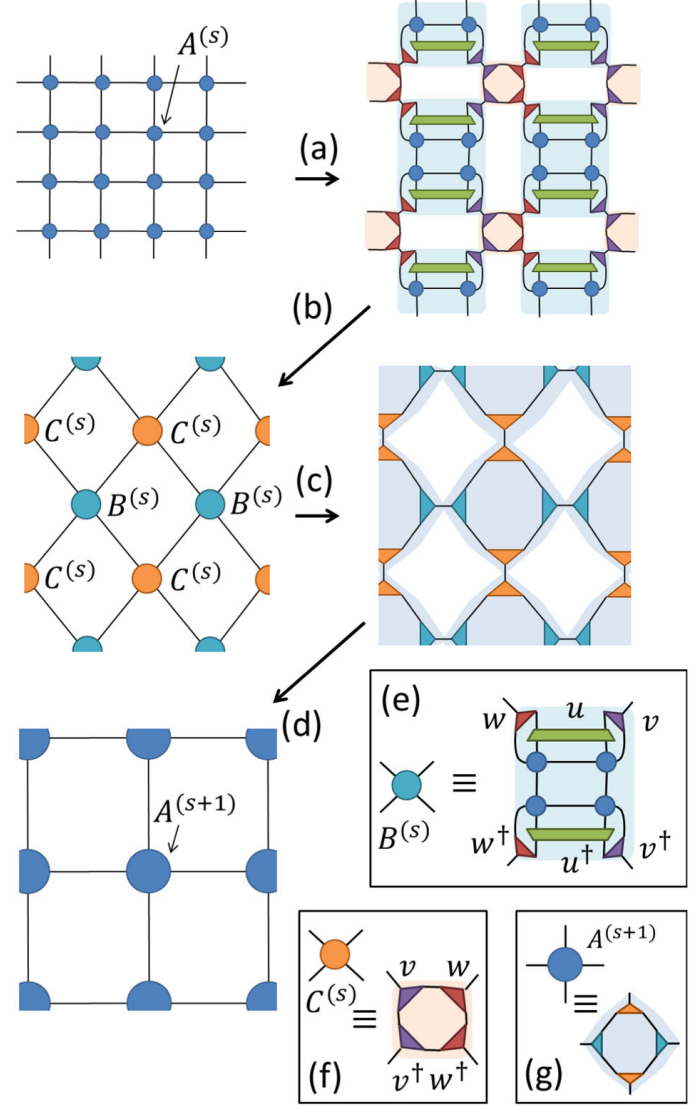

FIG. 2 (color online). (a)-(d) Steps of a TNR transformation to produce tensor $A^{(s+1)}$ from tensor $A^{(s)}$. In (a), the insertion of disentanglers $u$ and isometries $v$ and $w$ is made according to Fig. 1(b). Insets (e) $-(\mathrm{g})$ contain the definition of the auxiliary tensors $B^{(s)}$ and $C^{(s)}$ and the coarse-grained tensor $A^{(s+1)}$.

function $Z$ only contains short-range correlations (technically, this corresponds to a so-called corner double-line (CDL) tensor; see Ref. [11], Sec. B). If we set the disentanglers to be trivial, $u=I^{\otimes 2}$, then the coarse-graining transformation reduces to the TRG and fails to remove the short-range correlations. However, with a judicious choice of disentanglers $u$, these correlations are removed and an uncorrelated, trivial tensor $A^{\text {triv }}$ is produced $[11,28]$. Therefore, the role of disentanglers is to remove short-range correlations. Their action will be particularly important at criticality, where correlations are present at all length scales.

Example: Partition function of the $2 \mathrm{D}$ classical Ising model.-We consider the partition function

$$
Z=\sum_{\{\sigma\}} e^{-H(\{\sigma\}) / T}, \quad H(\{\sigma\})=-\sum_{\langle i, j\rangle} \sigma_{i} \sigma_{j}
$$

on the square lattice, where $\sigma_{k} \in\{+1,-1\}$ is an Ising spin on site $k$ and $T$ denotes the temperature. Recall that this model has a global $Z_{2}$ symmetry: it is invariant under the simultaneous flip $\sigma_{k} \rightarrow-\sigma_{k}$ of all of the spins. We obtained an exact representation for the tensor $A$ in Eq. (1) in terms of four Boltzmann weights $e^{\sigma_{i} \sigma_{j} / T}$,
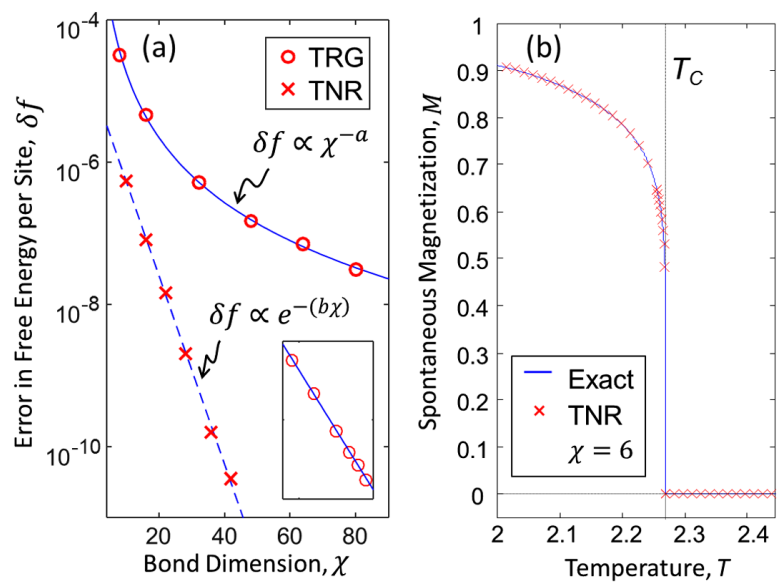

FIG. 3 (color online). Benchmark results for the square lattice Ising model on a lattice with $2^{39}$ spins. (a) Relative error in the free energy per site $\delta f$ at the critical temperature $T_{c}$, comparing the TRG and TNR over a range of bond dimensions $\chi$. The TRG errors fit $\delta f \propto \chi^{-3.02}$ (the inset displays them using log-log axes), while TNR errors fit $\delta f \propto \exp (-0.305 \chi)$. Extrapolation suggests that the TRG would require bond dimension $\chi \approx 750$ to match the accuracy of the $\chi=42$ TNR result. (b) Spontaneous magnetization $M(T)$ obtained with TNR with $\chi=6$ [30]. Even very close to the critical temperature, $T=0.9994 T_{c}$, the magnetization $M \approx$ 0.48 is reproduced to within $1 \%$ accuracy.

$$
A_{i j k l}=e^{\left(\sigma_{i} \sigma_{j}+\sigma_{j} \sigma_{k}+\sigma_{k} \sigma_{l}+\sigma_{l} \sigma_{i}\right) / T},
$$

which corresponds to having one tensor $A$ for every two spins, and a tensor network with a $45^{\circ}$ tilt with respect to the spin lattice; see Figs. 1(a) and 1(b). We actually built our starting tensor $A^{(0)}$, with bond dimension $\chi=4$, by joining a square block of four tensors $A$ together. We then applied up to 18 TNR transformations to a system made of $N=2^{18} \times$ $2^{18}$ tensors $A^{(0)}$ or, equivalently, $2 \times 4 \times N$ Ising spins.

Figure 3(a) shows the relative error $\delta f$ in the free energy per site $f \equiv \log (Z) /(8 N)$, at the critical temperature $T_{c} \equiv 2 / \ln (1+\sqrt{2}) \approx 2.269$, for both the TRG and TNR as a function of the bond dimensions $\chi$ [29]. The TRG error decays polynomially, while the TNR error is reduced exponentially, showing a qualitatively different behavior and implying that significantly more accurate results can be obtained with TNR. Figure 3(b) shows the spontaneous magnetization $M(T)$ as a function of temperature $T$ obtained with TNR for $\chi=6$. Even close to $T=T_{c}$, we see remarkable agreement with the exact solution.

However, the most significant feature of TNR is revealed in Fig. 4, which shows, as a function of the scale $s$, the spectrum of singular values of tensor $A^{(s)}$ when regarded as a matrix $\left[A^{(s)}\right]_{(i j)(k l)}$. Figure 4(a) considers the critical point, $T=T_{c}$, and shows that under TNR, the spectrum of $A^{(s)}$ quickly becomes independent of the scale $s$. This has two major implications. On the one hand, it is a strong evidence that $A^{(s)}$ itself has converged to a fixed-point tensor $A^{\text {crit }}$ (up to small corrections; see Ref. [11], Sec. A for details), thus 

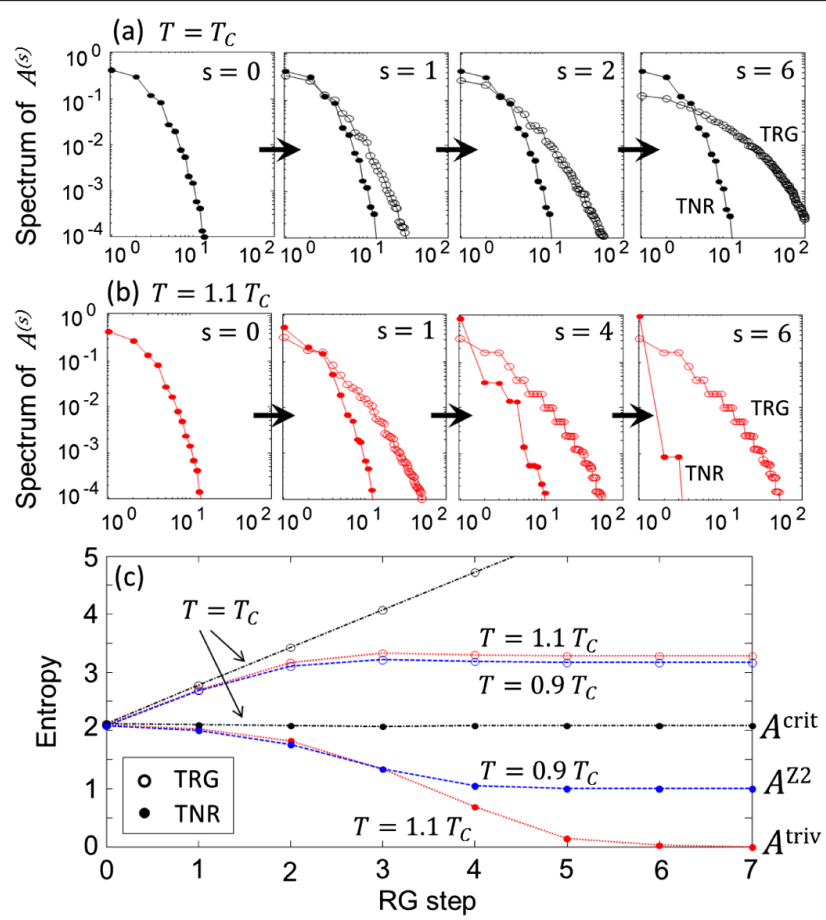

FIG. 4 (color online). (a) Singular values $\lambda_{\alpha}$ of the matrix $\left[A^{(s)}\right]_{(i j)(k l)}$ obtained after $s$ RG steps [31] using TNR (the filled circles) or the TRG (the empty circles) for the 2D Ising model at critical temperature $T_{C}$. (b) Singular values for $T=1.1 T_{C}$. (c) Plot of the von Neumann entropy $-\sum_{\alpha} \tilde{\lambda}_{\alpha} \log \left(\tilde{\lambda}_{\alpha}\right)$ of the (normalized) singular values of tensors $\left[A^{(s)}\right]_{(i j)(k l)}$ obtained with the TRG (the empty circles) or TNR (the filled circles).

recovering the characteristic scale invariance expected at criticality. On the other hand, it implies that the bond dimension $\chi$ required to maintain a fixed, small truncation error $\|\delta\|$ is essentially independent of scale, and thus so also is the computational cost. That is, we have obtained a computationally sustainable RG transformation. In sharp contrast, the spectrum generated by the TRG develops a growing number of large singular values as we increase the scale $s$, indicating that the tensor is not scale invariant. The bond dimension (and thus the computational cost at constant truncation error) grows rapidly with scale. This growth is caused by the accumulation of short-range correlations at each iteration and pinpoints the breakdown of the TRG at a critical point; see also Ref. [11], Sec. C.

Figure 4(b) considers a slightly larger temperature, $T=1.1 T_{c}$. Now, TNR generates a flow towards the trivial fixed-point tensor $A^{\text {triv }}$, characterized by just one nonzero singular value, which represents the infinite temperature, disordered phase. As expected of a proper RG scheme, for any $T>T_{c}$ the flow is to the same trivial fixed-point tensor $A^{\text {triv }}$. In contrast, for any $T>T_{c}$, the TRG generates flows to a fixed-point tensor that depends on the initial temperature $T$. In other words, failure to remove some of the shortrange correlations implies that (RG irrelevant) microscopic information has been retained during coarse graining, contrary to the spirit of the RG flow. For $T<T_{c}$ (not shown), we obtain a similar picture as for $T>T_{c}$. However, now each eigenvalue in the spectrum has degeneracy 2, and TNR flows to a new fixed-point tensor $A^{Z_{2}} \equiv A^{\text {triv }} \oplus A^{\text {triv }}$ corresponding to the $Z_{2}$ symmetry breaking, ordered phase-the $Z_{2}$ spin flip symmetry acts on $A^{Z_{2}}$ by exchanging its two copies of $A^{\text {triv }}$. Finally, Fig. 4(c) uses the entropy of the spectrum as a function of scale to visualize the RG flow towards one of the three fixed-point tensors: the ordered $A^{Z_{2}}$ for $T<T_{c}$, the critical $A^{\text {crit }}$ for $T=T_{c}$, and the disordered $A^{\text {triv }}$ for $T>T_{c}$.

Outlook.-We have proposed TNR, a coarse-graining transformation for tensor networks that produces a proper RG flow in the space of tensors, and we have demonstrated its performance for 2D classical partition functionsincluding the explicit recovery of scale invariance at the critical point. When applied to the Euclidean path integral of the 1D quantum Ising model (after suitable discretization in the imaginary time direction), it produced results similar to the ones described above. The approach can also be used to compute the norm $\langle\Psi \mid \Psi\rangle$ of a $2 \mathrm{D}$ quantum many-body state encoded in a projected entangled pair state [32].

TNR borrows its key idea - the use of disentanglers - from entanglement renormalization [18,19], the coarse-graining scheme for many-body wave functions that led to the multiscale entanglement renormalization ansatz (MERA) [33]. The two approaches turn out to be deeply connected: when applied to the Euclidean path integral of a Hamiltonian $H$, TNR produces a MERA for the ground and thermal states of $H$ [34].

We thank Z.-C. Gu and X.-G. Wen for clarifying aspects of their TEFR approach [7]. G. E. is supported by the Sherman Fairchild Foundation. G. V. acknowledges support by the John Templeton Foundation and the NSERC. The authors also acknowledge support by the Simons Foundation (the Many Electron Collaboration). Research at Perimeter Institute is supported by the Government of Canada through Industry Canada and by the Province of Ontario through the Ministry of Research and Innovation.

*evenbly@caltech.edu

† gvidal@perimeterinstitute.ca

[1] L. P. Kadanoff, Scaling laws for Ising models near $T_{c}$, Physics (Long Island City, N.Y.) 2, 263 (1966); E. Efrati, Z. Wang, A. Kolan, and L. P. Kadanoff, Real-space renormalization in statistical mechanics, Rev. Mod. Phys. 86, 647 (2014).

[2] K. G. Wilson, Renormalization group methods, Adv. Math. 16, 170 (1975); The renormalization group: Critical phenomena and the Kondo problem, Rev. Mod. Phys. 47, 773 (1975).

[3] S. R. White, Density matrix formulation for quantum renormalization groups, Phys. Rev. Lett. 69, 2863 (1992); Density-matrix algorithms for quantum renormalization groups, Phys. Rev. B 48, 10345 (1993). 
[4] M. Levin and C. P. Nave, Tensor Renormalization Group Approach to 2D Classical Lattice Models, Phys. Rev. Lett. 99, 120601 (2007).

[5] H.-H. Zhao, Z.-Y. Xie, Q.-N. Chen, Z.-C. Wei, J. W. Cai, and T. Xiang, Renormalization of tensor-network states, Phys. Rev. B 81, 174411 (2010).

[6] Z.-Y. Xie, H.-C. Jiang, Q.-N. Chen, Z.-Y. Weng, and T. Xiang, Second Renormalization of Tensor-Network States, Phys. Rev. Lett. 103, 160601 (2009).

[7] Z.-C. Gu and X.-G. Wen, Tensor-entanglement-filtering renormalization approach and symmetry protected topological order, Phys. Rev. B 80, 155131 (2009).

[8] H. C. Jiang, Z. Y. Weng, and T. Xiang, Accurate Determination of Tensor Network State of Quantum Lattice Models in Two Dimensions, Phys. Rev. Lett. 101, 090603 (2008); Z.-C. Gu, M. Levin, and X.-G. Wen, Tensor-entanglement renormalization group approach as a unified method for symmetry breaking and topological phase transitions, Phys. Rev. B 78, 205116 (2008); B. Dittrich, F. C. Eckert, and M. Martin-Benito, Coarse graining methods for spin net and spin foam models, New J. Phys. 14, 035008 (2012).

[9] Z.-Y. Xie, J. Chen, M. P. Qin, J. W. Zhu, L. P. Yang, and T. Xiang, Coarse-graining renormalization by higher-order singular value decomposition, Phys. Rev. B 86, 045139 (2012); A. Garcia-Saez and J. I. Latorre, Renormalization group contraction of tensor networks in three dimensions, Phys. Rev. B 87, 085130 (2013); S. Wang, Z. Y. Xie, J. Chen, B. Normand, and T. Xiang, Phase transitions of ferromagnetic Potts models on the simple cubic lattice, Chin. Phys. Lett. 31, 070503 (2014).

[10] As originally described by Levin and Nave in Ref. [4], the breakdown of the TRG at criticality refers to the fact that (1) the computational cost to obtain a fixed accuracy in local expectation values increases as a critical point is approached, and (2) while the TRG flows to some fixed-point tensor off criticality, this is no longer the case at criticality [4]. However, the TRG is still useful for studying critical systems; see, e.g., Refs. [4,7,11].

[11] See Supplemental Material at http://link.aps.org/ supplemental/10.1103/PhysRevLett.115.180405, which includes Refs. [12-17], for further details on a number of aspects of tensor network renormalization, including an analysis of the scale-invariant fixed points yielded by the approach, and a comparison with other tensor coarsegraining approaches for both noncritical and critical systems.

[12] R. N. C. Pfeifer, G. Evenbly, and G. Vidal, Entanglement renormalization, scale invariance, and quantum criticality, Phys. Rev. A 79, 040301(R) (2009); G. Evenbly and G. Vidal, in Strongly Correlated Systems: Numerical Methods, edited by A. Avella and F. Mancini, Springer Series in SolidState Sciences Vol. 176 (Springer, New York, 2013), Chap. 4.

[13] G. Evenbly, R. N. C. Pfeifer, V. Pico, S. Iblisdir, L. Tagliacozzo, I. P. McCulloch, and G. Vidal, Boundary quantum critical phenomena with entanglement renormalization, Phys. Rev. B 82, 161107(R) (2010); G. Evenbly and G. Vidal, Algorithms for Entanglement Renormalization: Boundaries, Impurities and Interfaces, J. Stat. Phys. 157, 931 (2014).
[14] B. Swingle, Entanglement renormalization and holography, Phys. Rev. D 86, 065007 (2012); H. Matsueda, M. Ishihara, and Y. Hashizume, Tensor network and a black hole, Phys. Rev. D 87, 066002 (2013); T. Hartman and J. Maldacena, Time evolution of entanglement entropy from black hole interiors, J. High Energy Phys. 05 (2013) 014; J. MolinaVilaplana and J. Prior, Entanglement, tensor networks and black hole horizons, Gen. Relativ. Gravit. 46, 1823 (2014); A. Mollabashi, M. Nozaki, S. Ryu, and T. Takayanagi, Holographic geometry of cMERA for quantum quenches and finite temperature, J. High Energy Phys. 03 (2014) 098; G. Evenbly and G. Vidal, Tensor network states and geometry, J. Stat. Phys. 145, 891 (2011); B. Swingle, Constructing holographic spacetimes using entanglement renormalization, arXiv:1209.3304; J. Haegeman, T. J. Osborne, H. Verschelde, and F. Verstraete, Entanglement Renormalization for Quantum Fields, Phys. Rev. Lett. 110, 100402 (2013); F. Pastawski, B. Yoshida, D. Harlow, and J. Preskill, Holographic quantum error-correcting codes: Toy models for the bulk/boundary correspondence, J. High Energy Phys. 06 (2015) 149; M. Miyaji and T. Takayanagi, Surface/state correspondence as a generalized holography, Prog. Theor. Exp. Phys. 2015, 073 B03 (2015); X.-L. Qi, Exact holographic mapping and emergent space-time geometry, arXiv:1309.6282; N. Bao, C. Cao, S. M. Carroll, A. Chatwin-Davies, N. Hunter-Jones, J. Pollack, and G. N. Remmen, Consistency conditions for an AdS/MERA correspondence, Phys. Rev. D 91, 125036 (2015).

[15] Z.-C. Gu and X.-G. Wen (private communication).

[16] P. Di Francesco, P. Mathieu, and D. Senechal, Conformal Field Theory (Springer, New York, 1997).

[17] H. W. J. Blöte, J. L. Cardy, and M. P. Nightingale, Conformal Invariance, the Central Charge, and Universal FiniteSize Amplitudes at Criticality, Phys. Rev. Lett. 56, 742 (1986).

[18] G. Vidal, Entanglement Renormalization, Phys. Rev. Lett. 99, 220405 (2007).

[19] G. Evenbly and G. Vidal, Algorithms for entanglement renormalization, Phys. Rev. B 79, 144108 (2009).

[20] In this Letter (as in Levin and Nave's TRG paper [4]), a fixed-point tensor is a tensor that is (explicitly) invariant under coarse graining; see also Ref. [11], Sec. A.

[21] TEFR contains an entanglement filtering step designed to simplify CDL tensors (see Ref. [11], Sec. B) by filtering out their short-range correlations [7]. The filtering step allows TEFR to recover the proper structure of noncritical RG fixed points - in contrast with the TRG [4], where for a given phase one obtains a continuum of fixed-point CDL tensors that only differ in short-range correlations. However, TEFR cannot simplify tensors near a critical point [22].

[22] X.-G. Wen (private communication).

[23] The gauge transformation is made such that the block of four $A$ tensors is manifestly invariant with respect to reflection along the horizontal axis, which is a convenient choice in order to preserve reflection symmetry in the network.

[24] An arbitrary $n$-point correlator will then be computed by first inserting $n$ operators in the tensor network of Eq. (1), and then conveniently adjusting its coarse graining. 
[25] The role disentanglers play in TNR, where they remove short-range correlations, is analogous to their role in the context of entanglement renormalization of ground state wave functions, where they remove short-range entanglement (hence the name) [18].

[26] One should take into account the local environment, as described in Ref. [5], when decomposing an auxiliary tensor $C^{(s)}$, which may otherwise possess a slowly decaying spectrum of singular values that cannot be accurately truncated.

[27] G. Evenbly, Algorithms for tensor network renormalization, arXiv: 1509.07484 .

[28] For the specific case of CDL tensors, the filtering step of TEFR [7] is also capable of eliminating the local correlations [21]. TNR removes short-range correlations both away from criticality and near or at a critical point.

[29] The bond dimension $\chi$ of the TNR calculation denotes the largest bond dimension used. In practice, different bond dimensions are used at different steps of the TNR coarsegraining transformation.
[30] The spontaneous magnetization is extracted from the coarse-grained spin operator, by analyzing the projection onto, e.g., the spin-up symmetry-breaking sector.

[31] We define a single RG step as that which maps the square lattice back into a square lattice with the same orientation, but one whose linear dimension has been reduced by a factor of 2. Note that this corresponds to two RG steps, as originally proposed in Ref. [4].

[32] F. Verstraete and J. I. Cirac, Renormalization algorithms for quantum-many body systems in two and higher dimensions, arXiv:cond-mat/0407066; F. Verstraete, J. I. Cirac, and V. Murg, Matrix product states, projected entangled pair states, and variational renormalization group methods for quantum spin systems, Adv. Phys. 57, 143 (2008).

[33] G. Vidal, Class of Quantum Many-Body States That Can Be Efficiently Simulated, Phys. Rev. Lett. 101, 110501 (2008).

[34] G. Evenbly and G. Vidal, Tensor network renormalization yields the multi-scale entanglement renormalization ansatz, arXiv: 1502.05385 . 